\title{
Changing the Properties of the CdTe/ZnTe Quantum Dots by in situ Annealing during the Growth
}

\author{
P. WojnAR ${ }^{a}$, G. KARCZEWski ${ }^{a}$, T. Wojtowicz ${ }^{a}$ \\ AND J. KOSSUT ${ }^{a, b}$ \\ ${ }^{a}$ Institute of Physics, Polish Academy of Sciences \\ al. Lotników 32/46, 02-668 Warsaw, Poland \\ ${ }^{b}$ ERATO Semiconductors Spintronics Project \\ al. Lotników 32/46, 02-668 Warsaw, Poland
}

\begin{abstract}
We present an attempt to control the properties of CdTe/ZnTe self-assembled quantum dots during their formation in the process of molecular beam epitaxy. Namely, the structures were in situ annealed at various temperatures and annealing times after the formation of quantum dots, before the deposition of a capping layer. Depending on the annealing parameters, the dots exhibit different optical properties which were studied by means of spatially resolved photoluminescence. From the analysis of these results, the information about relative changes of the average size and sheet density of quantum dots was extracted.
\end{abstract}

PACS numbers: 78.67.De, 78.55.Et

\section{Introduction}

Self-assembled semiconductor quantum dots (QDs) are one of the most frequently studied quantum structures, because of a common interest in fundamental physics of excitons confined in zero-dimensional environment and in view of their possible applications as a component of optoelectronic devices [1-3]. QDs based on III-V semiconductors are studied more intensively in comparison with their counterparts based on II-VI material systems, due to some difficulties in the fabrication of the latter structures. In $\mathrm{CdTe} / \mathrm{ZnTe}$ material system, the QD formation does not take place spontaneously despite a similar lattice mismatch as in commonly used InAs/GaAs system, but it is usually induced by an additional procedure such as exposing a thin strained $\mathrm{CdTe} / \mathrm{ZnTe}$ quantum well structure to a Cd flux at an enhanced substrate temperature $[4,5]$, or covering it with amorphous tellurium 
at a low substrate temperature and its subsequent desorption [6, 7]. The main advantage of the latter method is the possibility of in situ observation of the QDs formation by means of reflection of high energy electron diffraction (RHEED), similarly as for III-V compounds. The latter method has been applied in the present work.

In this work, we present an attempt to control the properties of CdTe/ZnTe quantum dots by means of the in situ annealing of the QD-ensemble before deposition of the cap layer. We show that there are two types of zero-dimensional structures in the ensemble: "regular" QDs formed as a result of the StranskiKrastanov growth mode transition and "quasi" 0D islands formed by potential fluctuations at the interfaces of the quasi-wetting layer. Depending on the annealing parameters the one or another type of these objects becomes more populated.

\section{Experimental}

All samples under consideration are grown by the same procedure. Firstly, a $4 \mu \mathrm{m}$ thick CdTe buffer layer is deposited on $001 \mathrm{GaAs}$ substrate. After the CdTe buffer, a $1 \mu \mathrm{m}$ thick ZnTe barrier layer is grown. The quantum dots are formed by deposition of 6 monolayers of CdTe which are covered with an amorphous tellurium layer at a low substrate temperature [6, 7]. After thermal desorption of the Te layer at $350^{\circ} \mathrm{C}$, a clear phase transition from a $2 \mathrm{D}$ to a $3 \mathrm{D}$ surface is observed in the RHEED pattern. The transition is a direct proof of the formation of the self-assembled QDs. Next, an annealing process is applied in situ in the molecular beam epitaxy (MBE) deposition chamber just after the formation of the QDs and before capping them with $100 \mathrm{~nm}$ of ZnTe. The surface is exposed at tellurium flux during the annealing, in order to avoid strong desorption of CdTe from the surface and to avoid a possible incorporation of impurities. In the case of annealing at relatively high temperatures $\left(\approx 380^{\circ} \mathrm{C}\right)$ for times longer than $10 \mathrm{~min}$, the RHEED image shows that the surface becomes two-dimensional again. This indicates that the surface roughness does not exceed 3 monolayers (ML) and points to a considerable desorption of CdTe QDs from the surface.

\section{Results and discussion}

In Fig. 1a, three normalized photoluminescence (PL) spectra from samples annealed for 5,10 , and $30 \mathrm{~min}$ at a relatively low temperature of $310^{\circ} \mathrm{C}$ are presented. One observes that an additional peak at $2.0 \mathrm{eV}$ appears and its intensity increases with an increasing annealing time. The total intensity of the emission does not change much. This effect can be explained in terms of a ripening process which takes place in the uncapped QD-ensemble and results in formation of dots with relatively large height. The PL line at the high energy part of the spectrum $(2.2 \mathrm{eV})$ does not change the spectral position by annealing. We attribute this line to the emission from the quasi-wetting layer (i.e., not necessarily continuous $2 \mathrm{D}$ layer). As it will be shown by our further measurements, this emission consists 


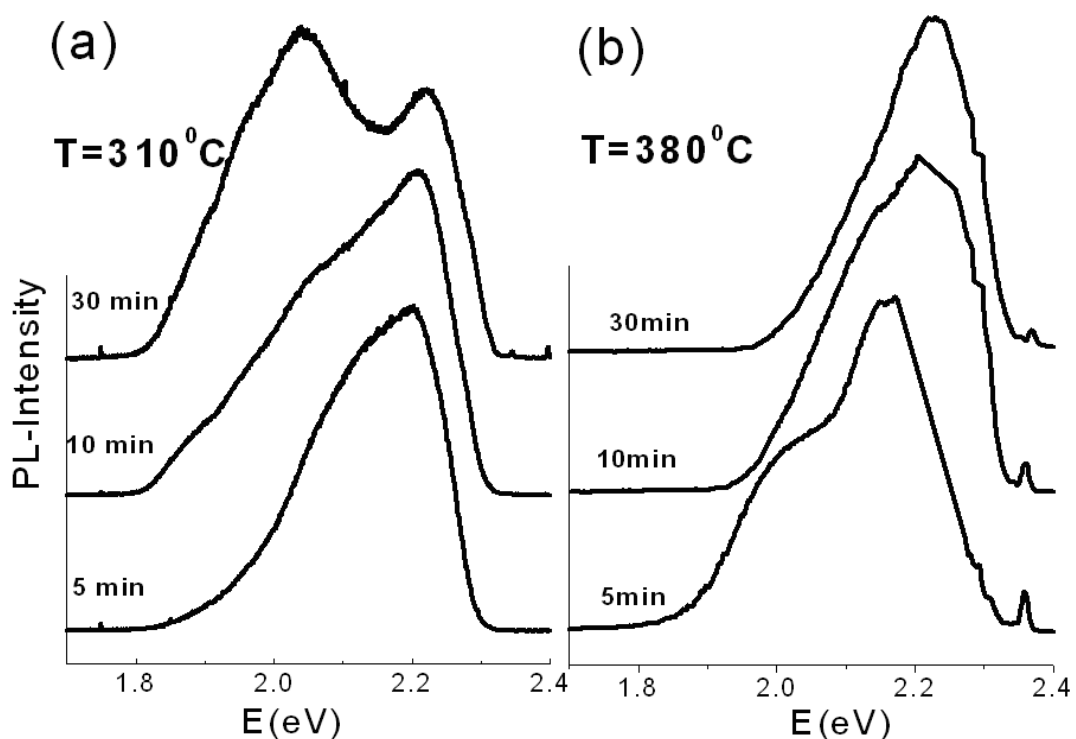

Fig. 1. Normalized photoluminescence spectra from 6 samples containing CdTe/ZnTe quantum dots annealed at various temperatures and times: (a) $310^{\circ} \mathrm{C}$ during 5,10 , and $30 \mathrm{~min}$, (b) $380^{\circ} \mathrm{C}$ during 5,10 , and $30 \mathrm{~min} . T=10 \mathrm{~K}$, non-resonant excitation $442 \mathrm{~nm}$ He-Cd laser.

of zero-dimensional flat islands formed as a result of potential fluctuations at the interfaces of a wetting layer.

This interpretation is supported by annealing at higher temperatures, e.g., $380^{\circ} \mathrm{C}$. One observes that the photoluminescence band shifts towards higher energies (Fig. 1b). After $30 \mathrm{~min}$ annealing, the emission appears mostly in the energy range corresponding to the high energy line visible in Fig. 1a. Let us note again that the surface observed by means of electron diffraction for this annealing time becomes two-dimensional. This can be explained in terms of desorption of the significant part of the CdTe deposit from the surface, which is additionally supported by a considerable decrease in the total PL intensity. Therefore, we conclude that the PL emission remaining after annealing for $30 \mathrm{~min}$ at $380^{\circ} \mathrm{C}$ must be related either to a 2D wetting layer, or to flat "quasi" 0D islands, whose height does not exceed $3 \mathrm{ML}$ and whose presence cannot be detected by RHEED. In order to exclude one of these possibilities, the temperature dependence of the PL intensity and micro-PL measurements are performed.

In Fig. 2, the temperature dependence of the total PL intensity from $\mathrm{CdTe} / Z n T e$ QDs structures annealed during $30 \mathrm{~min}$ at $380^{\circ} \mathrm{C}$ (Fig. 2a), at $310^{\circ} \mathrm{C}$ (Fig. 2c), and from the "as grown" sample (Fig. 2b), are presented. The main process which leads to the thermally induced decrease in the PL intensity is related to the thermal escape of heavy holes from the quantum structure [5] and depends strongly on the quantum confinement of the carriers. Thermal activation energy 


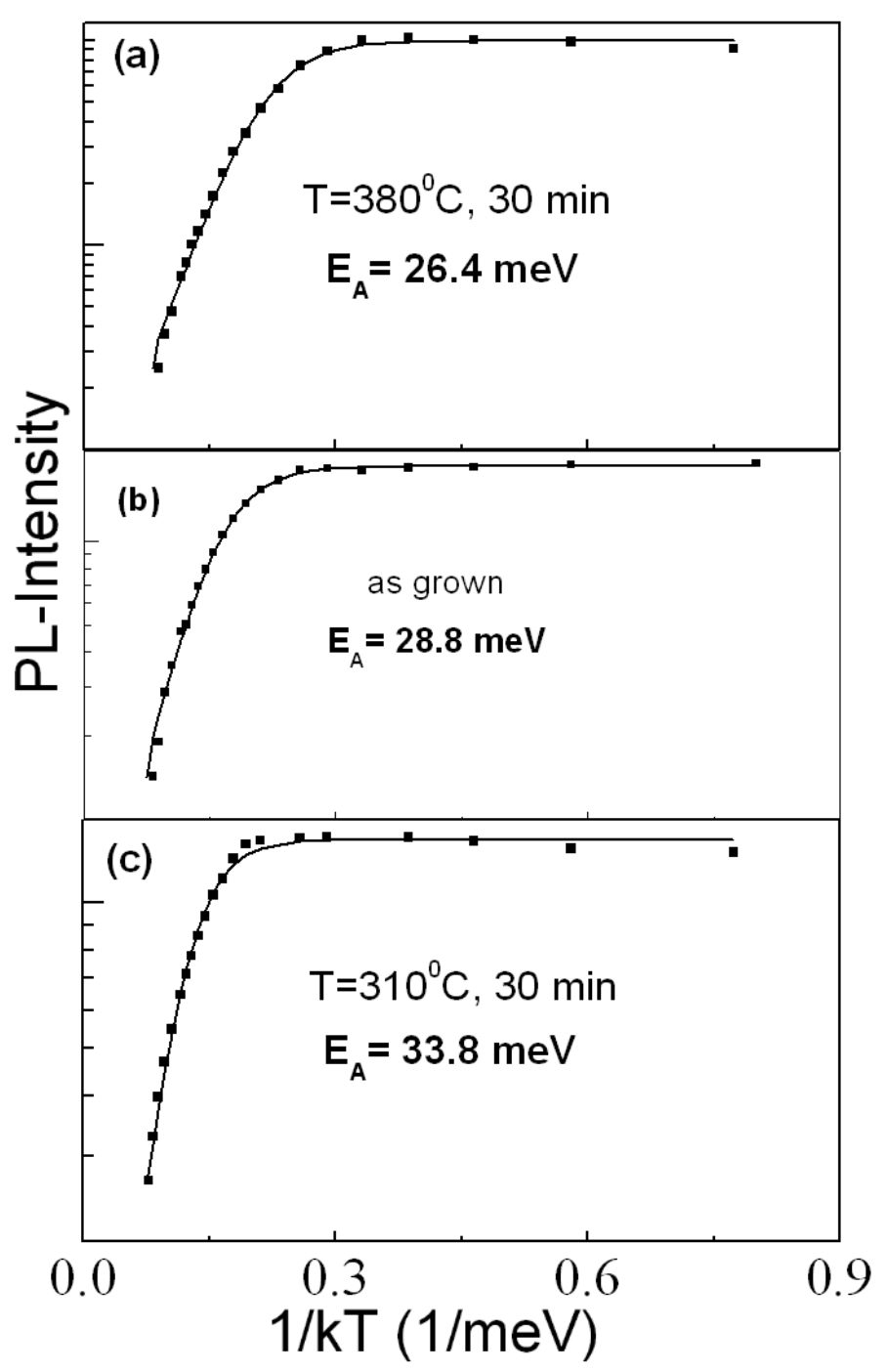

Fig. 2. Arrhenius plot of the PL intensity vs. temperature ranging from $20-200 \mathrm{~K}$ from which thermal activation energy of non-radiative processes $-E_{\mathrm{A}}-$ is determined for 3 samples with CdTe/ZnTe QDs: (a) annealed $30 \mathrm{~min}$ at $380^{\circ} \mathrm{C}$, (b) as grown sample, (c) annealed $30 \mathrm{~min}$ at $310^{\circ} \mathrm{C}$.

for non-radiative processes is determined using the following equation:

$$
I=\frac{I_{0}}{1+C \exp \left(-E_{\mathrm{A}} / k T\right)} .
$$

$E_{\mathrm{A}}$ is the thermal activation energy of non-radiative processes, $C$ is the ratio of non-radiative to radiative transition probabilities, $I_{0}$ - proportionality factor describing PL intensity at the lowest temperatures. 
The values of the thermal activation energy amounts to $26.4 \mathrm{meV}, 28.8 \mathrm{meV}$ to $33.8 \mathrm{meV}$ for the samples: annealed at $380^{\circ} \mathrm{C}$, as grown one, and annealed at $310^{\circ} \mathrm{C}$, respectively. They are all considerably larger than reported for $2 \mathrm{D}$ structures based on CdTe/ZnTe material system (12-17 meV) [8]. Thus we conclude that all the observed PL emissions are associated with zero-dimensional objects. In particular, the high energy line at $2.2 \mathrm{eV}$ from Fig. 1 is associated to the emission from flat $0 \mathrm{D}$ islands.

Moreover, the thermal activation energy from the "quasi" 0D structures, $E_{\mathrm{A}}=26.4 \mathrm{meV}$ (Fig. 2a), is considerably smaller than in the case when the PL emission consists also from "regular" QDs (Fig. 2c), $E_{\mathrm{A}}=33.8 \mathrm{meV}$ in the sample annealed at $310^{\circ} \mathrm{C}$. This can be explained by a significantly larger quantum confinement and larger dots sizes of "regular" QDs.

In the case of micro-PL measurements, when the excitation spot is of the order of $2 \mu \mathrm{m}$, one observes that the whole PL emission from all samples splits into a series of sharp lines with the spectral width of the order of $100 \mu \mathrm{eV}$ associated to the emission from individual QDs, see Fig. 3. This effect is particularly pronounced
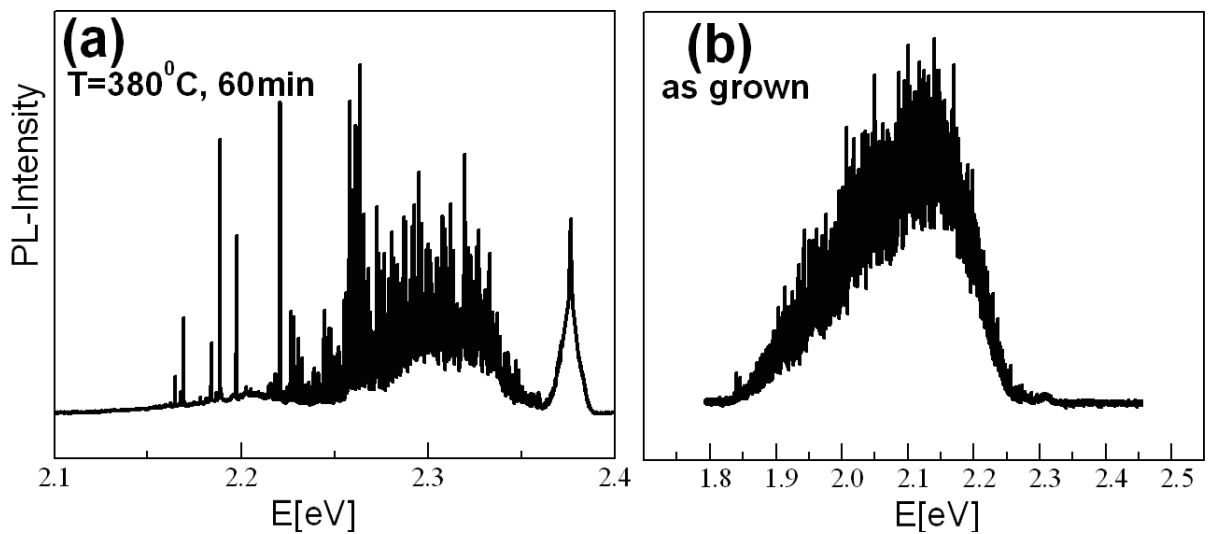

Fig. 3. Micro-photoluminescence from CdTe/ZnTe QDs: (a) annealed for $60 \mathrm{~min}$ at $380^{\circ} \mathrm{C}$, representing a sample with low density of QDs, (b) as grown, representing sample with high density of QDs. Excitation spot diameter $2 \mu \mathrm{m}, 442 \mathrm{~nm} \mathrm{He}-\mathrm{Cd}$ laser, $T=$ $10 \mathrm{~K}$.

in the case of the sample annealed for $60 \mathrm{~min}$ at $380^{\circ} \mathrm{C}$ with an average emission at $2.3 \mathrm{eV}$, Fig. 3a, which confirms the 0D origin of this line. Generally, a considerably better resolution of individual PL lines is observed for QDs annealed at relatively high temperatures in comparison to "as grown" samples, Fig. 3b. We explain this finding as a result of a decrease in the density of QDs due to desorption of the dots from the surface. 


\section{Conclusion}

Summarizing, an in situ annealing of self-assembled QDs CdTe/ZnTe leads to significant changes of the average QDs size and their density. The annealing at relatively low temperatures is responsible for ripening process, i.e., the formation of large dots. The annealing at high temperatures results in desorption of CdTe QDs from the surface, whereas only 0D islands formed as a result of potential fluctuation of the wetting layer remaining on the surface.

\section{Acknowledgments}

This work was partly supported by the SANDiE Network of Excellence, and by the Ministry of Science and Higher Education (Poland) under the grant N202 $05032 / 1187$.

\section{References}

[1] Y. Kong, Y. Ding, R. Yang, Z.L. Wang, Science 303, 1348 (2004).

[2] A. Alim, V.A. Fonoberov, A.A. Balandin, Appl. Phys. Lett. 86, 053103 (2005).

[3] C. Erwin, L. Zu, M.I. Haftel, A.L. Efros, T.A. Kennedy, D.J. Norris, Nature (London) 436, 91 (2005).

[4] G. Karczewski, S. Maćkowski, M. Kutrowski, T. Wojtowicz, J. Kossut, Appl. Phys. Lett. 74, 3011 (1999).

[5] S. Maćkowski, G. Karczewski, T. Wojtowicz, J. Kossut, A. Heiss, Appl. Phys. Lett. 78, 3884 (2001).

[6] F. Tinjod, B. Gilles, S. Moehl, K. Kheng, H. Mariette, Appl. Phys. Lett. 82, 4340 (2003).

[7] F. Tinjod, I.C. Robin, R. Andre, K. Kheng, H. Mariette, J. Alloys Comp. 371, $63(2004)$.

[8] S. Maćkowski, G. Karczewski, F. Kyrychenko, T. Wojtowicz, J. Kossut, Thin Solid Films 367, 210 (2000). 